Cipango Cahiers d'études japonaises

19 | 2012

Le Japon et le fait colonial II

\title{
Introduction aux recherches philosophiques de
} Fukuzawa Yukichi

La révolution chez Fukuzawa et la notion de jitsugaku

Maruyama Masao

Traducteur : Isabelle Lefebvre

\section{OpenEdition}

1 Journals

Édition électronique

URL : https://journals.openedition.org/cipango/1724

DOI : $10.4000 /$ cipango. 1724

ISSN : 2260-7706

\section{Éditeur}

INALCO

\section{Édition imprimée}

Date de publication : 30 octobre 2012

Pagination : 191-217

ISBN : 978-2-85831-204-7

ISSN : 1164-5857

Référence électronique

Maruyama Masao, « Introduction aux recherches philosophiques de Fukuzawa Yukichi », Cipango [En ligne], 19 | 2012, mis en ligne le 15 mai 2014, consulté le 30 juin 2021. URL : http://

journals.openedition.org/cipango/1724; DOl : https://doi.org/10.4000/cipango.1724

Ce document a été généré automatiquement le 30 juin 2021.

\section{(c) (i) (8)}

Cipango est mis à disposition selon les termes de la Licence Creative Commons Attribution - Pas d'Utilisation Commerciale 4.0 International. 


\section{Introduction aux recherches philosophiques de Fukuzawa Yukichi}

La révolution chez Fukuzawa et la notion de jitsugaku

\section{Maruyama Masao}

Traduction : Isabelle Lefebvre

\section{NOTE DE L'ÉDITEUR}

Maruyama Masao, « Fukuzawa ni okeru « jitsugaku » no tenkai. Fukuzawa Yukichi no tetsugaku kenkyū josetsu » 福沢に於ける「実学」の展開、福沢諭吉の哲学研究序説, mars 1947, in Maruyama Masao shū 丸山真雄集, vol. XVI, Tōkyō, Iwanami shoten, (1997), 2004, p. 108-131. Les notes de bas de page rédigées par la traductrice sont précédées de la mention NDT, en revanche, les notes de Maruyama sont indiquées sans cette mention.

\section{Préface}

1 On affirme que Fukuzawa Yukichi est le Voltaire du Japon et il n'est pas exagéré de dire que, chez nous, parler d'Aufklärung ${ }^{1}$, c'est parler de Fukuzawa. À partir du moment où, juste après la restauration de Meiji, avec la réalisation consécutive d'œuvres comme Seiyō jijō (La situation de l'Occident), Sekai kuni zukushi (Les pays du monde), Seiyō ryo annai (Guide de voyage en Occident) ou Gakumon no susume (L'Appel à l'Étude) ${ }^{2}$, Fukuzawa a rapidement pris la position de leader intellectuel de la nouvelle époque, à partir de ce moment et jusqu'à nos jours, c'est-à-dire durant environ quatre-vingts ans et tout au long de cette période, sa pensée a systématiquement été associée dans les esprits à la demande d'Aufklärung. 
2 Dans le numéro 362 de la revue Kokumin no tomo (L'ami de la nation) ${ }^{3}$, Ōnishi Hajime écrivait :

Alors que le courant de pensée de l'Aufklärung, surgi comme par surprise, n'est pas encore parvenu à son terme, on se préoccupe déjà de réaliser un retour au passé et on tient comme la pire des choses qui puissent arriver que de briser le lien qui nous unit à ce passé. Je pense qu'il est trop tôt pour être nostalgique de la tradition, que si l'oubli du passé est à craindre plus que tout, c'est à tort que l'on considère que c'est par loyauté envers l'État qu'on doit y rester attaché ; ô combien ce malentendu a fait tomber jusqu'à présent le monde de notre éducation dans l'obstination et l'étroitesse.

(...) Face à ces considérations, je ne peux que m'attrister de ce que le courant de l'Aufklärung consécutif à la Restauration de Meiji ne progresse avec plus de vigueur et n'adopte aujourd'hui un esprit davantage révolutionnaire. En voyant comment notre bon vieux maître Fukuzawa a maintenu intact jusqu'à aujourd'hui ce courant de pensée de l'Aufklärung, et en constatant tout particulièrement ces derniers temps à quel point il s'efforce de l'encourager à voix haute, on ne peut que ressentir de la sympathie pour lui. Hélas, quelle extrémité n'a-t-on pas atteinte dans la perte de tempérament ou d'esprit de révolution et de rénovation en s'attachant aux distinctions historiques et à la continuité de la tradition. On doit déplorer l'affaiblissement des revendications intemporelles que ce soit au sein de la littérature, de la philosophie, de la religion, de la morale, du devoir ou des sentiments humains.

3 Cette protestation s'élevait en 1897, une année où la vie brillante de Fukuzawa allait bientôt s'éteindre. Un peu plus tard dans le temps, dans les premières années de l'ère Taishō, Tanaka Ōdō $\overline{4}^{4}$ avertissait :

La signification du Mouvement pour l'Aufklärung [Keimōe e no undō] n'est pas encore suffisamment assimilée par nos compatriotes. (...) Tant qu'ils ne l'ont pas bien comprise et n'y ont pas été initiés, ils ne parviendront véritablement, quoiqu'ils fassent, à former un peuple civilisé.

Dans le premier chapitre de son livre Fukuzawa Yukichi, Tanaka Ōdō arbore comme un slogan l'expression «Revenons à Fukuzawa». Par le désastre qu'est maintenant la défaite, il apparait en pleine lumière combien a été détourné de son but initial ce parcours de modernisation effectué depuis la restauration du Japon. Il semble que si l'on rappelle sur scène Fukuzawa Yukichi, c'est parce qu'il s'est développé une vive conscience de la nécessité de réapprendre sans préjugés ce qu'est la liberté moderne et qu'il a été perçu comme libéral, utilitariste et individualiste.

Il y a bien sûr une raison évidente au retour à Fukuzawa chaque fois que l'Aufklärung redevient un thème d'époque. À travers son œuvre et le mouvement pour l'éducation, sa vie entière a été vouée à l'éducation du peuple et, indubitablement, sa position idéologique s'est inscrite dans le prolongement du courant d'idées de l'Aufklärung, au sens large de cette expression. Mais d'un autre point de vue, il est indéniable que le fait que son nom ait toujours été associé à l'Aufklärung s'est révélé un obstacle à l'examen minutieux et sans réserve des fondements philosophiques de sa pensée.

La critique impitoyable de la réalité historique est ce qui a marqué les esprits et reste l'essentiel du contenu de la pensée de l'Aufklärung, mais, en contrepartie, on ne s'est pas intéressé aux fondements méthodiques de sa critique. À ce propos, cette pensée manque souvent de réflexivité et la philosophie de l'Ancien Régime qu'elle tente de surmonter se révèle paradoxalement plus fine. La pensée de l'Aufklärung comporte en revanche cette capacité à démasquer le conservatisme enfoui sous cette finesse. Cela explique que lorsque Fukuzawa a émergé en tant que philosophe-type de l'Aufklärung, 
les gens ont été dépossédés de leur clairvoyance par la pertinence et le caractère éblouissant de sa critique concrète du phénomène pathologique de la société japonaise et, tout naturellement, ne prêtèrent pas attention au paradigme profondément inhérent à sa critique. C'est de cette façon que la pensée de Fukuzawa a été globalement assimilée à celle des autres penseurs de l'Aufklärung de la première période de l'ère Meiji et qu'on l'a ainsi catalogué par de vagues dénominations telles que " rationalisme », « utilitarisme » ou " positivisme franco-anglais ».

7 Si Fukuzawa ne fait apparaître nulle part en tant que tel son point de vue sur les valeurs et sur la connaissance, c'est bien parce qu'il n'est pas un philosophe au sens strict du terme. Toutefois, si on lit dans le détail toute son œuvre, on y reconnaît et une façon propre d'estimer les valeurs et une cohérence dans sa façon de voir les choses. Par ailleurs, à aucun moment on ne peut réduire sa pensée à la vision commune des penseurs de l'Aufklärung qui lui étaient contemporains car sa pensée est extrêmement singulière. Si l'on ne peut dire qu'avoir catégoriquement rangé Fukuzawa comme simple rationaliste de l'éducation, réaliste ou encore utilitariste, soit tout à fait une erreur, il en a cependant résulté l'effacement complet de son caractère singulier. Je veux mettre particulièrement l'accent sur le fait que c'est précisément cette attitude même de Fukuzawa qui fonde l'esprit d'indépendance et la dignité qu'il a mis en avant. En remontant jusqu'à ce fondement pour Fukuzawa de l'esprit libre, on s'aperçoit qu'il avait une compréhension bien plus profonde qu'on n'a voulu longtemps le penser et on peut de mieux saisir la portée actuelle des problèmes qu'il a soulevés.

Pour comprendre la méthode fondamentale de pensée qui était la sienne, je voudrais tout d'abord ici prendre pour paradigme son point de vue sur les études. Approfondir ce point est une condition préalable indispensable pour se placer dans une juste perspective par rapport à sa pensée, parce qu'il me semble que le malentendu souvent commis à son égard provient d'une mauvaise compréhension de sa conception des études.

\section{Première partie}

9 Il est très souvent reconnu que Fukuzawa a amené une révolution par rapport à la manière traditionnelle de penser les études. Ce n'est pas un hasard si l'œuvre à laquelle il doit sa renommée a pour titre Gakumon no susume (L'Appel à l'étude). Ce livre était précisément la proposition d'un monde complètement nouveau d'études. Mais le problème réside dans le sens à donner à ce tournant révolutionnaire. A-t-il vraiment apporté un changement de direction?

Sa conception devenue célèbre des études est formulée dans le premier tome de Gakumon no susume $e^{5}$.

Les études, ce n'est pas connaître des caractères chinois complexes, lire des textes anciens difficiles à interpréter ou encore composer des poèmes traditionnels et écrire des vers chinois, ce n'est pas formuler des phrases qui ne portent aucun fruit dans le monde. Toute cette littérature contribue certes à réjouir les cœurs et élever les esprits, mais elle n'est pas aussi profitable que l'affirment depuis les temps reculés les confucianistes et les spécialistes des classiques japonais.

Aussi loin que l'on remonte dans le temps, on trouve peu de lettrés des études chinoises (kangakusha) habiles à gérer leur maison, et peu de citadins qui gèrent habilement leurs affaires et écrivent des waka. C'est pourquoi certains citadins ou paysans de bon sens, en voyant leurs enfants s'appliquer avec ardeur aux études, se 
mettent à craindre dans leur cœur de parents que le prix à payer n'en soit la prospérité de leur maison. Ce n'est pas une crainte vaine. Après tout, c'est le signe que ces études, en s'éloignant de la réalité, ne sont d'aucun usage quotidien. On doit reporter de telles études de peu d'utilité pratique et accorder la priorité à celles utiles au quotidien ordinaire des hommes.

11 Ainsi Fukuzawa a mis en avant, par rapport à l'étude vide et détournée de la vie des classiques chinois, et celle, désœuvrée, des règles de composition, les sciences pratiques proches de l'usage quotidien ordinaire des hommes. C'est précisément par cette position qui a été la sienne que pour la première fois a été envisagée la possibilité de se libérer d'études reposant sur une vie de parasite dépendant du travail d'autrui. Et qu'ensuite on a pu considérer que les vraies études étaient celles qui s'inscrivaient au sein même d'une vie reposant sur le principe " travailler par soi-même pour manger ». Cette prise de conscience est loin d'être sans importance. Cependant, le risque de faire perdre de vue une autre révolution inhérente à la conception des études chez Fukuzawa, et plus essentielle chez lui, était contenu dans la primauté accordée à ces phrases, dans le fait que jitsugaku ${ }^{6}$ soit devenu un terme en vogue, et que les études qu'il a proposées n'aient été diffusées qu'en tant qu'elles concernaient les affaires. C'est principalement à partir de la lecture de ce passage qu'a pu germer une vision commune de l'ensemble de ses études comme d'un utilitarisme ou d'un réalisme vulgaire.

Si les revendications du jeune Fukuzawa ne s'étaient appliquées purement et simplement qu'au « simple aspect pratique des études » ou à «l'union des études et de la vie quotidienne", une telle manière de penser n'aurait jamais été très révolutionnaire. Sur ce point Fukuzawa aurait indubitablement été critique, mais non révolutionnaire. Qu'il abhorre les spéculations conceptuelles vides, qu'il aspire à ce que les études soient au service de la vie pratique (comme je l'ai mentionné plus haut, un problème se pose par rapport à la signification concrète de cette expression), c'est une attitude traditionnelle, peut-on dire, dans les conceptions des Japonais ${ }^{7}$. On dit en effet que le désintéressement pour les études théoriques éloignées des nécessités pratiques est justement la caractéristique des études en Orient ${ }^{8}$. Au sein de l'école Teishugaku ${ }^{9}$ se trouvait beaucoup plus de systémacité abstraite que dans la pensée confucéenne. C'est cette école qui a introduit le terme jitsugaku et a rendu florissant son usage. Son enseignement a été importé au Japon au cours de la première période des Tokugawa et peu de temps après que l'on se soit enorgueilli de son succès florissant, son caractère conceptuel et discursif fut mis en évidence par Kumazawa Banzan ${ }^{10}$ et Yamaga Sokō ${ }^{11}$. Ce dernier pensait par exemple que ceux qui ne se conformaient pas au juste chemin de l'étude,

quand bien même s'appliqueraient-ils à accorder leurs actes et leurs paroles, se donneraient-ils des règles de conduite et apprendraient-ils par cœur une multitude de mots et de phrases, il est clair qu'ils se disperseraient loin du chemin de l'étude de la Voie des Saints. ${ }^{12}$

Il a ainsi rejeté (ibid.)

l'érudit [moji no gakusha 文字の学者], grand ignorant du quotidien. Les lettres et les études ne sont pas nécessaires au vrai confucianiste, il s'agit pour lui d'accepter ce qu'il doit faire le jour même et de s'en satisfaire.

Ce qu'il définissait ainsi comme véritable étude, il l'a précisément nommé jitsugaku. Comparons avec la notion de jitsugaku de Fukuzawa. L'une est l'attaque menée contre des études qui ne sont que "récitations de mille mots et dix mille vers, ignorance du quotidien »; l'autre, contre des études qui se résument à l'«apprentissage de 
caractères difficiles et la lecture de textes anciens difficiles à comprendre, mais qui ne sont d'aucune utilité quotidienne ». biens et à se détruire eux-mêmes, dans sa revendication pour une union des études à la vie économique quotidienne, Baigan se trouve dans la droite ligne de ce qui sera plus tard la thèse de Fukuzawa. On peut donc dire que si Fukuzawa fut exceptionnel, ce n'est ni pour ses efforts et actions rejetant des études oisives éloignées de la vie, ni pour avoir proposé des études réellement utiles au quotidien, et pas non plus pour avoir rattaché les études à la vie du peuple en les libérant du monopole qu'en avait la classe dominante. On a compris que son orientation a toujours été en continuité avec la tradition des études de l'Ancien Régime. Par conséquent, si on ne saisit la pensée de Fukuzawa que par ces aspects-là, la notion d'études pratiques se tient tout au plus aux fondements du pragmatisme occidental et ne va pas au-delà d'une pensée au cœur de l'esprit de son époque. Même s'il s'y trouve le développement de ce qui n'était qu'à l'état d'immanence, il n'y a ni saut vers les autres ni rupture avec le passé.

e véritable tournant révolutionnaire de la notion d'études pratiques chez Fukuzawa ne réside ni dans l'union des études et de la vie ni dans l'affirmation même de l'utilité effective des études; c'est bien plutôt dans sa façon de relier les études et la vie que se situe la question essentielle. Et le tournant fondamental que comporte cette façon de les relier provient du changement de structure fondamentale des études. Chercher le sens de ce changement nous permettra de comprendre l'esprit des études pratiques chez Fukuzawa. 


\section{Deuxième partie} études à une époque donnée, dans une société donnée, dépend des valeurs fondamentales qui y sont fixées par rapport au monde et à la vie. Par conséquent, à l'inverse, on peut connaître les changements mêmes de manière de vivre des gens à travers les changements du domaine central de leurs études. En ce sens, en voyant lesquelles chez lui ont été considérées comme l'origine des études-types et en les comparant avec celles de l'Ancien Régime, on peut voir clairement ce qui est révolutionnaire chez Fukuzawa.

21 Dans son autobiographie ${ }^{17}$, Fukuzawa s'exprime de la façon suivante :

Depuis longtemps, il y a réellement une grande différence quant à la rapidité du progrès en Orient et en Occident. Bien qu'il y ait, d'un côté comme de l'autre, un enseignement moral, des débats économiques ainsi que points forts et points faibles tant dans le monde des lettres que dans le monde martial, en ce qui concerne l'enrichissement du pays, le renforcement de l'armée et les conjonctures nationales, les pays orientaux se situent sans nul doute dans l'ensemble en dessous des pays occidentaux relativement au bonheur optimal que peut atteindre la plus grande partie de la population. Sur cette question, si on compare confucianisme en Orient et civilisation occidentale, il manque du côté oriental deux points, à savoir, les mathématiques ${ }^{18}$ en ce qui concerne le monde matériel, et l'autonomie [dokuritsu 独 立] en ce qui concerne le monde de l'esprit. En toute affaire humaine, que ce soit récemment pour l'édification de notre pays, ou à plus long terme, pour l'humanité en tant que telle, les hommes ne peuvent absolument pas échapper aux mathématiques ${ }^{19}$. Au Japon, il n'a pas été suffisamment pris en compte qu'il n'y avait d'autre position où se tenir que celle de l'autonomie. Ce n'est pas ainsi que nous pourrons nous aligner sur les pays occidentaux puissants.

Autrement dit, Fukuzawa a cherché et trouvé le secret de l'immobilité des sociétés orientales dans le manque conjoint d'esprit d'autonomie et de connaissance mathématique. Il apparaît de plus en plus clairement combien les deux sont liés l'un à l'autre. Le problème qui nous occupe ici concerne le point central des études européennes, point central qu'il a désigné sous le terme de "mathématiques ». Quand il parle de mathématiques, il s'agit, au sens strict du terme, de la physique-mathématique moderne, autrement dit, il se rapporte au système mécanique formé par Newton (ailleurs, le terme de physique est utilisé simplement comme il le fait dans Zoku fuku ō hyaku wa 続福翁百話, Suite des cent propos du vieux Fukuzawa, 1882) : «la différence entre les études orientales et les études occidentales réside seulement dans le fait de prendre ou de ne pas prendre la physique pour base ». Chez Fukuzawa, cela constituait, pour ainsi dire, l'étude des études, la règle de toute étude, ou encore les études préparatoires : «pour entreprendre de suivre des études chez nous, à Keiō gijuku, il faut se concentrer principalement sur la physique, qui est préparation à toutes les matières » (《Butsurigaku no yōyō » 物理学之要用, [Ce qui est nécessaire aux études de la physique], 1897, Euvres complètes, vol. viII). Or, il va sans dire que, face à cette position de la physique, ce qui a occupé dans l'Ancien Régime une position centrale dans l'enseignement, c'est l'étude du gouvernement de sa propre maison, autrement dit, l'éthique. Là, les études sont « oshie » (Enseignement), les études de l'Enseignement sont ce que, originellement, doivent être les études. Et cet Enseignement est excellent, il n'est autre que celui de la « Voie ». 

déplacée de la morale vers la physique? Il va de soi qu'en aucun cas il ne s'agit d'un trivial et simple matérialisme consistant à remplacer, au centre de la vie humaine et du monde, les valeurs de l'esprit par celles de la matière. Il serait également erroné de penser que l'on a simplement déplacé le centre d'intérêt des études d'un point situé entre les principes moraux et la société vers le monde de la nature. Si l'on peut dire qu'au sein des études de l'Ancien Régime, dans une certaine mesure, il y avait également une métaphysique, il y avait aussi une physique. Les néo-confucéens aimaient à répéter qu'il fallait «rechercher le principe, approfondir un arbre, une plante ». D'un autre côté, je ne pense pas que Fukuzawa ait déterminé l'objectif des études davantage par rapport aux sciences de la nature que par rapport aux sciences humaines. La devise « indépendance et amour-propre » (dokuritsu jison 独立自尊) que Fukuzawa mettait en avant n'était pas autre chose qu'un problème d'éthique. Au cours des dernières années de sa vie, le programme en vingt-neuf points dans lequel son disciple a résumé sa théorie de l'indépendance et de l'amour-propre a été renommé, selon ses instructions, Shūshin yōryō (Points essentiels de l'éthique). Pour Fukuzawa, la question de la modernisation de notre pays a été abordée avant tout comme une recherche de "l'esprit» de la civilisation. Ne doit-on pas chercher le thème fondamental de l'Ébauche d'une théorie de la civilisation (Bunmeiron no gairyaku, 1875) dans cet avertissement à l'égard du mouvement d'ouverture de la civilisation qu'était aussi «le rejet de l'adoption, inhérente à son esprit » de la civilisation matérielle, «forme externe de la civilisation » ? Le fait qu'il ait pris la physique comme forme originelle des études n'était pas une dépréciation de "l'éthique» ou de «l'esprit», mais bien au contraire la prémisse fondamentale d'une nouvelle éthique et d'un nouvel esprit. Ce qui a aiguisé son intérêt, plus que les sciences naturelles elles-mêmes ou tous les fruits qu'elles ont apportés, c'était ce que doit fondamentalement être l'esprit humain qui a produit les sciences naturelles modernes. C'est bien le même esprit humain qui est au fond de l'éthique, de la politique, de l'économie ou de l'art moderne. Il n'y a pas d'antagonisme entre les études pratiques fondées sur l'éthique et les études pratiques fondées sur la physique, il faut revenir fondamentalement à l'antagonisme entre ce cœur de l'esprit qui engendre l'étude orientale de la Voie et celui qui engendre l'étude de la physique-mathématique moderne.

\section{Troisième partie}

Le fait que l'étude de l'éthique en ait été la forme primordiale, comme je l'ai dit plus haut, ne traduisait pas au sein des écoles de l'Ancien Régime un manque ou une insuffisance de connaissance de la nature. Le problème tient à ce que la nature n'est pas détachée des valeurs morales, et qu'un jugement éthique est continuellement introduit dans le phénomène naturel. La nature s'oppose à l'homme, elle n'est pas pensée comme extérieure, mais bien plutôt comme fondamentalement spirituelle. En même temps que 
la nature est spiritualisée, par son objectivation, l'esprit est rendu à la nature et il en résulte une indissociabilité des deux termes. Tournons-nous vers deux auteurs qui en ont rendu compte.

Le ciel est naturellement en haut, la terre est naturellement en bas. Une fois les positions du haut et du bas déterminées, celle du haut est noble, celle du bas est vile. Là où règne un ordre des principes de la nature, on doit le connaitre à la vue du haut et du bas. Il en va de même du cœur des êtres humains. Quand le noble et le vil, assimilés au haut et au bas, restent à leur place, les principes humains sont justes. S'ils sont justes, le pays est bien gouverné ; Hayashi Razan 林羅山, Keiten daisetsu 経典題説 (Discours doctrinal, 1667) ${ }^{20}$.

Le ciel en haut et la terre en bas, chacun a sa position propre; là se situe la racine essentielle de la morale. Depuis le soleil, la lune et les corps célestes, jusqu'au vent, à la pluie, au givre et à la neige, aux herbes et aux arbres, aux animaux et aux plantes, toutes les choses extériorisent une forme et une couleur, elles ont chacune leurs limites, elles ont toutes leur ordre cyclique. Un caractère supérieur ou inférieur vient caractériser progressivement chaque chose du ciel et de la terre. C'est cela « l'ordre du ciel et de la terre ». Les hommes saints ont formulé les règles en respectant cet ordre. Les règles respectant cet ordre sont les principes régnants; Kaibara Ekiken 貝原益軒, Go jō kun 五常訓 (Cinq préceptes usuels, 1711).

Si l'on compare ces deux discours, on s'aperçoit facilement d'une évidente similitude dans leur façon de progresser. Ce qui est visé ici est fondamentalement en relation avec l'ordre social issu des distinctions hiérarchiques. Cette base provient en même temps d'une analogie avec le monde de la nature. L'ordre social trouve le fondement de sa légitimité dans la correspondance avec une cohérence apparue dans les phénomènes naturels. Parce que cet ordre est conforme à celui de la nature, il est observé exactement comme un ordre naturel. Mais on doit sérieusement réfléchir au fait qu'on peut en réalité déceler dès l'origine les valeurs sociales au sein de la nature sur laquelle on doit fonder l'ordre social. Le fait que le ciel soit en haut et la terre en bas forme l'ordre du ciel et de la terre. Un ordre juste ne se maintient que lorsque l'homme est lui aussi lié au bien de la même façon que dans une relation hiérarchique. C'est pourquoi le principe selon lequel n'est maintenu un ordre juste que lorsque l'homme est lui aussi lié au Bien de la même façon que dans une relation hiérarchique, ne peut, même si l'on fait complètement abstraction de la naïveté d'une connaissance identifiant le rapport entre le ciel et la terre à un rapport hiérarchique, être reconnu autrement qu'en le rattachant à un rapport hiérarchique estimé comme une relation spatiale entre le haut et le bas. L'idée du positionnement social n'est pas fondée sur autre chose que sur la nature comprise à travers ce positionnement. Le fait que l'on ne perçoive pas comme telle cette tautologie témoigne plus que tout du caractère particulier de la conscience née de cette éthique des relations sociales.

C'est pourquoi il n'est pas tout à fait exact de dire que cette éthique s'exprime par le fondement de l'ordre social dans une analogie avec les phénomènes naturels. L'analogie renvoie d'ordinaire à l'hétérogénéité entre deux choses établies comme similaires, mais bien que cela soit ordinairement conscient, dans le cas qui nous occupe, elle n'est pas utilisée avec distinction du modèle humain et des lois de la nature, mais à l'inverse avec la déduction de quelque chose de fondamentalement commun. Les deux termes ne renvoient pas à autre chose qu'à la différenciation d'une même chose à l'origine en deux phénomènes distincts. Le renforcement mutuel du monde de la nature et de l'ordre social est possible par l'intermédiaire de ce quelque chose de fondamental qui gît au fond des deux. 
C'est ce quelque chose qui est aussi appelé Dō (Voie). La conscience de l'unité fondamentale entre la Voie qui se manifeste dans la nature (Voie du ciel) et la Voie qui domine les relations entre les hommes (Voie de l'homme) est le support de relations sociales hiérarchiques. L'enseignement produit par ces relations sociales est d'autant plus nécessairement enseignement de la Voie que cette conscience est le support de relations sociales hiérarchiques.

En ce sens, en tant que représentatif de l'enseignement de la Voie, et notamment dans le paradigme de l'enseignement qui y a, du point de vue de la morale, le plus préparé, à savoir l'école des Song, le confucianisme a admirablement mis en relief ce que sous l'Ancien Régime devaient être la nature, la société et les hommes. Autrement dit, pour le confucianisme, l'unité du ciel et des hommes a été fondée au sein de l'école des Song selon l'égalité du principe et du Faîte suprême (Taikyoku 太極); les hommes, la société et la nature sont traversés par le Faîte suprême. Le principe céleste (Voie du ciel) qui forme jusqu'au bout l'ordre cosmique, devient le principe vital inhérent à l'humanité, ce qui est objectivé en ordre social, c'est l'éthique des relations entre le maître et le vassal, le père et le fils, le mari et la femme, l'aîné et le cadet, les camarades. Par conséquent, parce que la norme de base de cet ordre social est donnée comme a priori inhérente à l'humanité, la manière d'être originelle de l'homme ne peut être ramenée qu'à un ordre social en tant que donnée objective. D'un autre côté, un tel ordre social est reproduit dans le mouvement perpétuel du fait qu'il est joint à l'ordre cosmique. L'homme est étroitement lié à la société, la société, étroitement liée à la nature. Ces trois ordres sont liés de façon admirable par le Faîte suprême ou Principe du ciel (l'Être ou la Voie du ciel), et, en réalité, la morale de la sincérité est, comme il est dit, fondamentalement donnée. Si l'on pense l'homme et la nature comme des valeurs, la morale de la sincérité est la morale fondamentale. Par conséquent, la recherche de la nature par laquelle on va jusqu'au bout du principe « un arbre, une plante » qui revient aussi à «donner le principe de chacune des choses » est quelque chose de conscient. Autrement dit, on trouve le seul et unique objectif des recherches naturelles dans le fait de prendre conscience du caractère moral fondamental inhérent aux choses naturelles en s'assurant davantage de la justesse innée de l'éthique (jin-gi-rei-chi-shin, les cinq vertus : bonté, justice, urbanité, sagesse et confiance) qui régit les lois humaines. La nature objective purement extérieure, indépendante des valeurs humaines, n'a pas eu de base sur laquelle se constituer. La physique n'a pu apparaître elle-même clairement qu'en tant que principe de la Voie. Dans la mesure où, au fond de cette orientation de pensée que l'on retrouve jusque dans l'école Teishu, se reflète à la fois le système social de l'Ancien Régime et une conscience universelle, une question fondamentale et commune à tous les courants de pensée ou écoles de cette époque se pose, celle du dépassement des différences doctrinales. Il est indubitable qu'une pureté telle que celle de l'esprit du confucianisme ne pouvait originairement restée intacte tout au long de l'époque des Tokugawa, de plus, comme nous l'avons indiqué, les spécificités des auteurs ont eu tendance à la corrompre progressivement ${ }^{21}$. L'étude de la Voie est un Idealtypus d'étude et on n'y a pas vu de révolution fondamentale par rapport à cet état de mélange subtil entre la physique et la morale, entre les lois de l'existence et les modèles de valeur. C'est cela même qui montre à quel point cette manière de penser s'enracine fortement et d'une façon singulière dans le système même de la société. Les relations de conditions et de classes agissent dans toute la société ; à partir du moment même où il naît, l'être humain a une place toute désignée et cet environnement le particularise comme une destinée. L'équilibre de la société repose sur la reconnaissance 
par tout être humain de sa position héréditaire comme d'un sort. La vie est la simple reproduction de la tradition et des coutumes, elle est tout aussi cyclique que le rythme des quatre saisons. Ici, ce n'est pas la société qui est portée par le sujet humain, mais, à l'inverse, c'est la manière d'être originelle des hommes qui est située dans la dépendance vis-à-vis de l'ordre de la société. La dépendance à l'égard d'un tel environnement donné a priori est une valeur, et s'en séparer est nécessairement une contre-valeur. Par conséquent, l'idéologie doit s'exprimer toute entière dans cette formule d'Ishida Baigan :

Si le malheur et le bonheur forment ensemble la destinée, allons dans le monde

tels que nous sommes avec les choses telles qu'elles sont, tel est l'Enseignement.

Il va naturellement de soi qu'à l'intérieur de ce système, ayant grandi dans le cadre de ces relations sociales, les hommes développent leur conscience en identifiant ordre social et ordre naturel. Il n'est pas non plus difficile d'en reconnaitre le revers: l'individu ne peut pas plus rester éloigné de son environnement social que des règles qui lui sont données par la société (celles d'être un vassal, un fantassin ou un marchand) et cette conscience ne se stabilise pas dans une union directe avec la nature. L'être humain prend conscience de son indépendance vis-à-vis de l'environnement social dans lequel il a noué son identité, dès lors qu'il s'apparaît à lui-même comme opposé directement et sans intermédiaire à la nature objective. L'indépendance vis-àvis de la société de l'individu est corrélative à celle de la nature et renvoie à une nature objective ou à la formation d'une nature purement extérieure de laquelle est ôtée toute valeur objective introduite par l'homme. En prenant conscience de sa subjectivité par rapport à son environnement, l'esprit, pour la première fois, se distancie des lois et des règles et se libère de la domination des principes de la physique et de la loi.

\section{Quatrième partie}

Il y a les choses et, ce faisant, il y a les principes, et non les principes et ce faisant les choses. Avant d'affirmer un jugement sans fondements, on explique d'abord rapidement le principe matériel, et on rencontre évidemment un principe physique dépendant d'un principe moral ; Bunmeiron no gairyaku, chapitre premier.

Le fait que cette affirmation de Fukuzawa ait tant fait date dans l'histoire des idées peut s'expliquer à partir de la seule description formulée plus haut. En ayant rejeté le caractère donné au préalable de l'ordre social, il a garanti l'indépendance objective de la physique. Les propos cités ci-dessus avaient directement pour objectif d'attaquer la théorie de l'école des Song qui prend pour a priori la relation du seigneur et du vassal, mais on peut dire aussi qu'ils constituaient la naissance de l'esprit physique. Fukuzawa avait toutefois clairement conscience que celle-ci n'était possible qu'avec une rébellion contre l'ordre des couches sociales. Qu'il ait pensé l'esprit de liberté et d'indépendance ainsi que la formation de la physique-mathématique comme centres de la civilisation européenne, révèle le degré de clairvoyance avec lequel il a pénétré la base de l'esprit moderne.

En Europe, la révolution la plus importante depuis la Renaissance s'est faite avec l'opposition de l'esprit et de la nature en tant que sujet intérieur et objet extérieur. Au Moyen Âge comme dans l'Antiquité, ces deux termes se sont interpénétrés chacun dans leur propre forme. La règle était alors celle d'une logique d'étages, la logique aristotélicienne de la forme et de la matière. Ensuite, en parallèle à cette logique, il s'en est ajouté une, propre à la philosophie scolastique, basée sur le système des couches 
sociales dans l'ordre de la société. L'appréhension moderne de la nature a aboli l'ordre aristotélicien des valeurs, enlevé à la nature toutes ses valeurs potentielles et s'est parachevée en un système de relations quantitatives réduites à des signaux et par conséquent purement mécanique. Mais le processus par lequel les valeurs ont été éliminées de la nature objective a simultanément été celui par lequel l'esprit-sujet s'est accaparé le monopole du sujet.

L'aliénation complète, par objectivation extérieure de la nature par l'esprit, s'est fait corrélativement à l'extériorisation de l'esprit s'orientant des couches sociales vers une mécanique consciente de son autonomie subjective. Le rapide essor des sciences naturelles modernes s'appuyant sur la physique newtonienne a été renforcé par l'éveil intense depuis Descartes de la raison subjective. Comme le dit Dewey, «par le fait de se soumettre théoriquement à la nature [la raison] a été esprit actif, vigoureux parce que maniant habilement l'expérimentation de la nature $»^{22}$.

Pour exprimer en un mot le caractère actif de cette raison moderne, on a eu recours à l'expression «esprit expérimental». C'est justement la notion d'expérience qui a distingué la connaissance moderne de la physique de celle du Moyen Âge. La raison, sans en rester à la simple conceptualisation de l'essence, y a l'initiative de reformer la nature à travers l'expérience et progresse infiniment vers de nouveaux domaines. De là, une maturation des fruits de la science moderne qui doit nous surprendre.

Pour l'exprimer autrement, que Fukuzawa ait fait de la physique un modèle idéal d'études revint à établir cet esprit expérimental comme noyau central de la méthode des études. En se basant sur la certitude selon laquelle «depuis le début des temps jusqu'à nos jours, on ne peut rien affirmer hors de l'examen du monde »; Bunmeiron no gairyaku, chapitre premier.

Fukuzawa a mis strictement en application cet esprit expérimental, non seulement dans le domaine des sciences naturelles, mais aussi jusque dans les différents domaines des sciences humaines tels que la vie politique ou la société. Les valeurs qui avaient été utilisées a priori en tant que traditions historiques, comme des dogmes particuliers, furent passées rigoureusement à l'examen de son esprit expérimental et révélèrent impitoyablement le mensonge de l'autorité. Avant lui, ni officiellement ni de fait, il n'y avait eu quelqu'un pour revendiquer de valeur absolue sans attendre la vérification de son usage pour la vie des êtres humains. Nous examinerons une prochaine fois cette manière fonctionnelle de penser, basée sur l'esprit expérimental. Je ne cherche rien d'autre ici qu'à montrer dans la philosophie de Fukuzawa le caractère indissociable de la doctrine de la physique et de l'esprit expérimental, la nécessité d'un continuel et indépendant maniement à travers l'expérience ainsi que la formulation claire de cette démarche expérimentale. C'est précisément sur ce dernier point que s'est située une rupture fondamentale entre deux rationalismes se tenant dans la position des sciences naturelles, celui de Fukuzawa et celui de Katō ${ }^{23}$ qui a perdu de vue le sujet humain luimême dans les liens nécessaires de la nature objective et du rationalisme.

\section{Cinquième partie}

37 Je pense que nous avons clarifié plus haut combien s'opposent les esprits respectifs au fondement de chacune des études pratiques qui pour noyau principal prennent l'une, « l'éthique », l'autre, la physique. Par conséquent, la différence fondamentale entre ces esprits doit également conduire à un tournant révolutionnaire dans la manière dont les 
études sont reliées à la vie et la pénètrent. Si les études de l'Ancien Régime consistent à connaître le principe de la Voie inhérente à la société naturalisée et à la nature qui a, pour ainsi dire, été socialisée, l'état d'âme idéal qui éveille à cette connaissance ne se trouve pas ailleurs que dans la parfaite union avec l'ordre de cette nature (société). C'est pourquoi il a été dit que "l'homme saint relie le ciel et la terre à ses vertus, le soleil et la lune à sa clarté, les quatre moments à l'ordre de sa vie» (Shuton.i / Zhou Dunyi 周敦匵, Taikyoku zusetsu 太極図説, Discours sur le Faîte suprême).

Ainsi, la hiérarchie des hommes vient se déterminer, chez l'homme saint, en tant que réponse aux déviations par rapport au degré de réunification avec cet ordre. Déranger l'éternel retour de l'ordre par le dépassement des limites de sa condition est le mal ultime. Le confucianisme dont on a dit qu'il était rationalisme de l'ordre (rationalismus der Ordnung ${ }^{24}$ ), en tant que principe premier, se trouvait aussi à l'aboutissement de l'école du cœur de l'époque d'Edo dans lesquelles il a été dit à propos du peuple : « aussi somptueusement revêtu qu'il soit, un cultivateur reste un cultivateur, un citadin reste un citadin et nul ne peut dépasser sa condition " (Seikaron ${ }^{25}$, premier volume), et par conséquent «ne pas tourner le dos à la juste direction des affaires sous le ciel, c'est cela l'étude du cœur ", formule qui se trouvait dans Yashinaigusa (Propos pour l'éducation). Ce qui régule donc l'attitude dans la vie quotidienne, c'est ici le principe d'adaptation à l'ordre en tant qu'environnement. Ne pas se séparer de l'environnement donné au moi n'est que le réalisme des études pratiques qui n'étaient pas autre chose qu'apprentissage de cette attitude de vie. Ici, approfondir la quotidienneté des études revient à se soumettre à des études orientées vers la vie quotidienne en tant qu'environnement objectif. Mais qu'en est-il chez Fukuzawa? Pour lui, la vie quotidienne n'a rien d'un environnement objectif.

Il n'y a pas de limite dans le développement de l'esprit humain. Ce qui développe l'esprit de l'être humain n'a pas de fins. Le mécanisme des changements de la nature n'est pas sans principes. Avec un esprit sans bornes et par l'approfondissement des principes déterminés, il n'y a finalement pas de distinction entre avoir une forme et ne pas en avoir et l'esprit humain peut comprendre tout en lui sans rien omettre des choses et faits sous le ciel et sur la terre; Bunmeiron no gairyaku, troisième chapitre.

39 Par la compréhension des principes de la physique, l'esprit humain cultive vigoureusement la nature objective et en la technicisant l'environnement du moi se forme objectivement.

D'un côté, par la considération de la réalité, les progrès de la théorie sont continuellement tirés en arrière, de l'autre, à l'inverse, la théorie progresse résolument vers sa subsumption dans le principe de l'étude des mille choses humaines. D'un côté, les études sont réellement adaptation à la réalité, de l'autre, elles sont transformation complète de la réalité.

$\mathrm{Si}$, en se penchant un peu sur la théorie, on la refuse comme une science vide, la façon d'être des études orientales consiste à ne pas oublier de revenir continuellement dans le domaine des études pratiques ; Nishi Shin.ichirō 西晋一郎, Tōyōrinri 東洋倫理 (Éthique orientale), 1934, p. 8.

41 Les études pratiques chez Fukuzawa prennent une direction tout à fait opposée : « même par rapport à chacune des plus petites choses du monde vulgaire, il ne doit y avoir aucun lieu où ne s'applique le principe des études » (« Keiō gijuku gakusei shoshi ni tsugeru », Déclaration aux étudiants de Keiō, Euvres complètes, vol. x). 
42 Parce que la réalité et la théorie passent toujours par l'intermédiaire de l'expérience, même dans le cas où l'application d'un principe a priori apparaît comme impossible, « il faut savoir garder à l'esprit que la recherche des principes est encore insuffisante » (Butsurigaku no yōyō, La nécessité des études de la physique), que l'on n'abandonne jamais en cours les principes moraux et que l'on ne peut faire de compromis facile avec la réalité. Par conséquent, les études pratiques de Fukuzawa, à l'opposé de l'attachement à la routine de la vie quotidienne ordinaire, étaient ce que continuellement on devait cultiver par la force de l'imagination qui entrevoit l'avenir par delà le quotidien. Ainsi, c'est bien paradoxalement la perpétuelle avancée vers des études «vides " placées au plus haut sous l'Ancien Régime qui garantit l'union la plus haute entre études et vie.

Si on voit, dans les moqueries de Fukuzawa citant le Kojiki pour évoquer les études par lesquelles on n'arrive même pas à gagner sa vie, ou certains propos extrêmes tels que «le but des hommes civilisés, c'est l'argent ${ }^{26}$, le développement de «l'Initiation à la façon de gagner de l'argent $\aleph^{27}$ et qu'on le résume à la branche principale de l'utilitarisme vulgaire des citadins d'Edo pour lesquels «les êtres humains sont semblables à des désirs qui auraient bras et jambes" (Ihara Saikaku), on ne peut absolument pas comprendre certaines des positions de Fukuzawautilisateur2014-05-15T09:46:00 ${ }^{28}$. En répétant et en insistant sur le fait que " pour faire des études, il faut en avoir le dessein et élargir celui-ci », Fukuzawa a donné un puissant avertissement à ne pas s'accrocher à des études dirigées vers la vie quotidienne. Ainsi, dans le dixième paragraphe de Gakumon no susume, il dit encore que, pour le développement des études:

parce que l'on ne peut compter ni sur l'actuel gouvernement vulgaire et sans éducation, ni sur une contribution telle qu'on puisse en tirer rapidement profit, il faut établir un institut de recherches et après avoir choisi cinq, six à dix savants, en faire un lieu qui pourvoit à leurs besoins tout au long de la vie en supprimant la racine de soucis autres que ceux des études.

En outre, en ce qui concerne les principes des études, il ne se demande pas «quel est le niveau de la personne à recruter, mais propose plutôt de lui donner les moyens de faire des études et de choisir quelque chose d'éloigné du véritable profit, comme on dit aujourd'hui» ("Jinsei no rakuji» [Les choses aisées de la vie], Euvres complètes, vol. XIV).

En réalité, le système de «la physique moderne » que Fukuzawa a recherché n'est en rien issu du caractère concret de la vie quotidienne qui adhère à l'environnement. Son utilitarisme réside dans sa compréhension bien plus profonde que celle de ses compatriotes de ce qui résulte d'un esprit libre qui a rejeté les entraves du monde sensible.

Quand l'adaptation à l'ordre objectif est devenue ce qui reçoit et forme l'avenir des hommes, il n'y a plus de frein au développement de l'opportunisme. Il ne s'agit pas de relier à des principes les conditions données au moi, mais au contraire de s'y conformer soi-même continuellement. Ici, l'expérience est comprise comme éternelle et le sujet formé à partir de l'objet n'est compris que passivement. Or, quelle que soit la quantité d'expériences de la vie quotidienne que l'on accumule, ce n'est pas à partir d'elles que se constituent les lois. Le sujet se constitue au sein de la réception passive de l'expérience par la simple objectivité, en ce que, comme il a été mentionné plus haut, il reconstruit positivement l'objet selon l'expérience réelle. 
Le concept moderne d'« expérience " contient ce moment actif et par conséquent, il génère non pas quelque chose d'ancien, mais une perspective future. Par le fait d'aller jusqu'à abstraire des lois à travers la réalité expérimentale qui adhère étroitement à l'expérience, la prévision et la mesure de l'avenir deviennent possibles. Fukuzawa a relevé les différences fondamentales entre la médecine traditionnelle chinoise et la médecine occidentale. Ce faisant, après avoir relevé l'appui de la seconde sur les principes de la vérité et l'origine de la première toute dans la pratique intensive du hasard, Fukuzawa montre que par conséquent, alors que la technique de la première comporte quelque chose d'universel et de cosmique, celle de la seconde se limite et trouve sa fin dans une époque pauvre en développement («Isetsu » [Discours sur la médecine], Guvres complètes, vol. xI), et ainsi le montre-t-il encore dans sa déclaration aux étudiants de Keiō (Euvres complètes, vol. xI). Fukuzawa critique l'admiration enthousiaste et sans réserve pour l'empirisme oriental et, à propos de la technique en Chine et au Japon par laquelle, par exemple, à partir du fer on fabrique un couteau, et par la taille d'arbres et de pierres on construit une maison, il note « la transformation artificielle d'un objet naturel en objet utile à la vie quotidienne» et ajoute que pour relever simplement l'opposition entre deux attitudes de vie discernables au fond des différences méthodologiques entre études orientales et études occidentales.

Tout peut reposer sur la physique, si l'on ne regarde que les résultats sans considérer les bases ni sans connaître les principes de vérité dans les études. Même si la technique s'est considérablement améliorée par la répétition d'exercices intensifs, on a seulement profité d'heureux hasards. Il faut savoir qu'on ne peut espérer de véritables améliorations et développements autrement, qu'il est insuffisant du point de vue de l'étude de ne compter encore que sur elle, malgré la beauté des résultats de cette technique.

C'est à un type complètement nouveau d'êtres humains qu'il destinait son éducation fondée sur les mathématiques et la physique ; des êtres humains mus par le rejet de son opportunisme "sans principes ni lois », basant et construisant continuellement la vie quotidienne sur la prévision et la mesure. Il se destina à l'éducation d'hommes combattant avec acharnement, exploitant sans limites le domaine d'une vie nouvelle à travers les essais et les erreurs.

\section{Sixième partie}

49 Je termine l'examen provisoire de ce que le tournant des études pratiques chez Fukuzawa a comporté de révolutionnaire pour l'esprit humain et pour finir je poserai un problème relié à cette question. Fukuzawa, comme il est mentionné ci-dessus, a considéré les sciences naturelles modernes non pas tant du point de vue de leurs résultats que de celui de l'esprit qui les engendre. On prend conscience de la liberté de la subjectivité humaine, et de la nature physique comme de deux pôles qui s'anticipent mutuellement. C'était certainement une vue profondément pénétrante du processus de formation de l'esprit moderne. Mais nous connaissons le processus par lequel, dans l'histoire de la pensée européenne, l'union de ces deux pôles s'est effondrée. Du symbole de la subjectivité qu'elle était, elle s'est muée en un mécanisme inquiétant qui a absorbé l'humain. L'homme a pris conscience de lui-même comme d'un corpuscule sans énergie dans l'enchaînement précis des causes et des effets au sein de l'Univers. La foi optimiste en l'infinité des progrès de la science de la période de l'Aufklärung s'est transformée en fatalisme et terrifiante désillusion. La théorie de 
l'évolution, en enseignant la sélection naturelle, a entériné cette désillusion. La science a étouffé la vie humaine au lieu de la libérer. Les hommes n'ont plus pu supporter la dureté de la nature mécanique arrachée aux différentes valeurs et significations intérieures, et finalement se sont enfuis dans un romantisme comme celui de Laskin ou réfugiés dans la glorification de la vie campagnarde qui fut celui de Tolstoï. Cela dépasserait le cadre de cet article que de s'interroger sur la justesse du changement de signification de cette image du monde donnée par les sciences naturelles. Ce qu'il faut ici déterminer, c'est si Fukuzawa a percé la nature du problème du scientisme qui constitue un pôle de cet esprit moderne, et s'il s'y est confronté. Fukuzawa distinguait dans la vie humaine le matériel (les choses qui ont une forme) et l'immatériel (celles qui n'en ont pas) - par exemple, la politique, l'économie, la littérature - et il n'a cessé de limiter, toujours provisoirement, l'application de la physique aux premières. Pourtant, tout en partant du fait que le domaine des secondes est radicalement dominé par la loi de la causalité, il semble avoir espéré qu'avec le développement de la sagesse humaine, les sciences naturelles pourraient un jour mesurer tous les domaines de la vie.

En ce sens, il a, sans mélange, été un enfant de l'Aufklärung. Il en partageait la foi, propre au rationalisme de ce mouvement, envers les progrès infinis de la raison et de la science. Mais, d'un autre côté, il ne faut pas oublier qu'il tenait des propos tels que :

Les hommes ordinaires sont dominés par deux fonctions, celle de la raison et celle du cœur, et la force du cœur, extrêmement puissante, entrave souvent le fonctionnement libre de la raison.

Ou encore :

Puisqu'il en est ainsi, tout en vivant dans le monde des sentiments humains, essayer de se conduire tout en se basant exclusivement sur le raisonnement calculant est quelque chose d'extrêmement triste et en réalité difficile pour un être humain; Tsūzoku dōtoku ron (Théorie de la morale populaire, Cuuvres complètes, vol. xI).

51 Face à cette puissante domination de l'irrationalité de la réalité l'approche réelle de Fukuzawa fut celle d'un progressisme exprimé dans des phrases telles que :

Il n'y a rien d'autre à faire que d'attendre que la société entière progresse, même si c'est petit à petit, en mélangeant le cœur humain et les principes de la logique mathématique. (Ibid.)

En cela, il est difficile de se soustraire au reproche d'extrême superficialité si l'on part de la position du rationalisme de l'Aufklärung. Or si l'on examine dans les détails sa critique de la société, un tel compromis avec la réalité irrationnelle se manifeste partout dans son œuvre. À la différence de beaucoup de penseurs de l'Aufklärung, Fukuzawa refuse de s'aligner sur les discours extrémistes et idéalistes vis-à-vis des changements sociaux ou politiques. Par rapport aux propos sur les droits et libertés du peuple, son célèbre discours sur l'harmonie entre le peuple et le gouvernement est l'expression la plus remarquable de ce refus. Jusqu'à quel point doit-on comprendre cette attitude réaliste de Fukuzawa? Si sa position se réduit au scientisme et au rationalisme de l'Aufklärung, cela signifie alors qu'il y a une certaine dissociation entre ses principes et son existence. En rejetant l'occasionisme, il n'a pas respecté la formule " vivons selon les principes et les lois ». Le fait même d'avoir un sentiment de tristesse à l'égard de l'attitude d'une vie qui dépend exclusivement du raisonnement calculant ne montre-t-il pas qu'il a dépassé la position du simple rationalisme abstrait ? S'il en est ainsi, une attitude réelle comme celle décrite ci-dessus n'est jamais un simple compromis, mais doit être interprétée selon ses « principes ». 
Ensuite, quel rapport le consentement à la réalité irrationnelle pourrait-il entretenir avec la doctrine physique? Autrefois, dans le réalisme de l'école du cœur cela n'a-t-il pas été suffisamment distingué ? Le fait que Fukuzawa ne soit pas complètement tombé dans le bourbier lugubre des théories scientifiques réglementées semble en relation avec cette question. Nous avons ici seulement introduit, avec peine, à l'intérieur de sa philosophie. L'objectif de cet article était d'ouvrir un chemin jusqu'à l'entrée. Je reprendrai la plume pour traiter du noyau central du problème.

\section{NOTES}

1. (NDT) Nous traduisons keimō 啓蒙, terme qui désigne les Lumières et l'instruction, par Aufklärung (éclairer, éclaircir) dans la mesure notamment où la plupart des intellectuels de l'époque Meiji préoccupés de la construction d'un État moderne s'intéressèrent surtout aux philosophies allemandes.

2. (NDT) Sur ces œuvres voir la notice introductive de Marion Saucier, Plaidoyer pour la modernité. Introduction aux œuvres complètes, op. cit., p. 18-20.

3. (NDT) Kokumin no tomo a été créée en 1887 par Tokutomi Sohō, et la société Min.yūsha 眠友者 (L'ami du peuple). Ouverte sur l'Occident, libérale, cette revue intéressait les intellectuels attirés par les idées de la Gauche. Avec Nihonjin 日本人 (Les Japonais), une revue s'inscrivant dans le nouveau courant nationaliste, L'ami de la nation est représentative de la presse de l'époque; Christiane Séguy, Le développement de la presse à l'époque Meiji et son rôle dans la modernisation du Japon, Paris, Publications Orientalistes de France, 1993, p. 38.

4. (NDT) Tanaka Ōdō 田中大堂 (1867-1932) a été le premier après des études à Chicago à présenter au Japon l'école philosophique américaine du pragmatisme fondée par Peirce, opposée aux idées abstraites et vagues et soucieuse des effets d'une théorie dans la pratique.

5. J'ai ajouté aux citations ci-dessous des signes de ponctuation, par commodité. En ce qui concerne les points à souligner dans les citations, là où il n'y a pas d'indications particulières, c'est l'auteur qui les a lui-même ajoutés.

6. (NDT) Sur ce terme, voir notre introduction et en particulier la note 1.

7. Sur ce point, voir par exemple Hasegawa Nyozekan 長谷川如是閑, Nihonteki seikaku 日本的性

格 (Le caractère japonais).

8. En général, en Orient, les études sont pratiques, et non pas simplement théoriques. La notion de jitsugaku a pour signification la recherche d'éclaircissement des problèmes de la vie réelle comme ceux de la religion, de la morale ou de la politique, du point de vue réel, et non pas la recherche qui nécessite de façon indispensable l'achèvement d'une structure théorique. Nishi Shin.ichirō, Tōyō rinri (Éthique orientale), p. 8.

9. (NDT) 程朱学, école fondée par Fujiwara Seika. Cf. introduction.

10. (NDT) 熊沢蕃山 (1619-1691). Cf. Jean-François Soum, Nakae Tōju et Kumazawa Banzan, Paris, Collège de France, IHEJ et CEJ, Paris, 2000.

11. (NDT) 山鹿素行 (1622-1685), fondateur de l'école des « études anciennes ».

12. (NDT) Yamaga Sokō, Haisho zanpitsu 配所残筆, (Dernier écrit sur un lieu d'exil), trad. Francine Hérail, Histoire du Japon, POF, 1986, p. 320-322.

13. (NDT) Yōmeigaku 陽明学, école Yōmei ou école de Wang Yangming. Cf. Maruyama Masao, Nihon seiji shisō shi kenkyū, trad. Jacques Joly, op. cit., p. 47, n. 1. 
14. (NDT) Mitogaku 水戸学, école qui commença à se développer avec son fameux projet de compilation de l'histoire nationale en 1657.

15. (NDT) Shingaku 心学, école au sein de laquelle étudier c'est connaître la nature en allant au fond du kokoro (心, cœur et esprit non différenciés).

16. (NDT) Ishida Baigan 石田梅岩 (1685-1744) fonda l'école Sekimon Shingaku 石門心学.

17. (NDT) Fukuō jiden 福翁自层, cf. La vie du vieux Fukuzawa racontée par lui-même, Marie-France Tellier (trad. et notes), Paris, Albin Michel, 2007.

18. (NDT) Sūri 数理, «le nombre et la raison" ou «les mathématiques ». Dans la mesure où Fukuzawa prend pour paradigme d'études pratiques la physique-mathématique, nous choisissons de traduire sūri par « mathématiques ».

19. (NDT) Les mathématiques, au sens spécifique du terme et distinct du calcul, renvoient aux principes de la réalité et l'humanité ne peut pas plus échapper à ces principes qu'à la réalité et ce, que le centre des études-types soit ou non la physique-mathématique.

20. (NDT) Hayashi Razan 林羅山, nom monastique Dōshun 道春 (1583-1657) poursuivit l'enseignement de Fujiwara Seika.

21. «Kindai jukyō no hatten ni okeru sōrai-gaku no tokushitsu nami ni sono kokugaku to no kanren»近代需教の発展に於ける徂徠学の特質並みにその国学との関連 (La particularité des études Sorai et leur relation avec les études nationales dans le développement du confucianisme moderne), Kokka gakkai zasshi (Journal de l'Association des sciences politiques et sociales), vol. 54, $\left.n^{\circ} 2,3,4,5\right)$ et « Kindai Nihon seiji shisō ni okeru “ shizen ” to “ sakui” ” 近代日本政治思想に於 けるく自然〉とく作為〉 (《La nature » et 《l'artifice» dans la pensée politique du Japon moderne), Kokka gakkai zasshi 国家学会䧱誌, vol.55, nº 7, 9, 12 ; vol. 56, nº 8 . Ici, l'auteur a examiné le processus de prise de conscience des limites de la pertinence du modèle de l'égalité « lois de la nature = modèle de la société = humanité » en même temps que sa continuité a été graduellement détruite dans le néo-confucianisme orthodoxe et que et chacun des termes est devenu conscient. C'est par rapport à une observation microscopique recherchant la germination de l'esprit moderne à la période des Tokugawa que l'exposé de cet écrit s'avère être une observation macroscopique de l'esprit de l'Ancien Régime. Ce qui a rendu représentatif cet esprit contenu dans les études Teishu n'est pas autre chose que ce qui en a clarifié le contraste avec l'esprit moderne. Par précaution, j'en ai touché un mot.

22. John Dewey, Reconstruction in Philosophy, 1920, p. 53.

23. (NDT) Katō Hiroyuki 加藤弘之 (1836 -1916), éducateur et homme d'état.

24. Max Weber, Confuzianismus und Taoismus (in Ges. Schrift zur Religionsoziologie, Marianne Weber S. 457).

25. (NDT) Seikaron 齐家論: Ken.yaku seikaron no susume 俟約斉家論のすすめ (Discours sur le gouvernement de la famille), ouvrage d'Ishida Baigan, paru l'année de sa mort (1744), sur les points fondamentaux de la Voie des marchands.

26. (NDT) Gakumon no shotoku wo katsuyō suru wa nanre no chii ni oite su nan kya 学問の所得を活用 するは何れの地位に於いてす何きや (À quel titre doit-on exploiter les revenus des études), Euvres complètes, vol. $\mathrm{x}$.

27. (NDT) 《Kane mōkaru denju» か站もうかるの伝授, Wakisaka gidō脇坂義堂 3, in Koizumi Yoshinaga 小泉吉永 (éd.), Sekimon shingakusho shūsei 石門心学書集成, vol. 10, Kuresu shuppan, 2013.

28. Par exemple, le professeur Watsuji Tetsurō, d'un point de vue où il considère L'appel à l'étude comme "une simple présentation populaire des idées utilitaristes et individualistes », affirme $q^{\prime}$ '« il est naturel que la pensée de Fukuzawa, en tant que ce dernier est pionnier de l'ère Meiji, ne soit - dans son essence - pas du tout différente d'Ihara Saikaku, Mitsui Takafusa et Ishida Baigan » (Gendai nihon to chōnin konjō. Zoku nihon seishin shi kenkyū 現代日本と町人根性、続日本 精神史研究 Le Japon moderne et l'esprit des citadins. Suite de l'étude sur l'histoire des mentalités au Japon). Prêtons attention au fait que s'il a souligné l'expression dans son essence 
c'est dans la mesure où il considère l'esprit des citadins comme de même essence que celui des bourgeois occidentaux. Watsuji se base sur le document de la famille d'Alberti en question, cité par Sombart (Tōyō bunka kenkyū, The Oriental Culture Review, n³ 3, mars 1947, Nikko sho.in 日光書 院). 